\title{
LA GESTIÓN DEL CONOCIMIENTO HERRAMIENTA DECISIVA EN LA GESTIÓN DE LOS RECURSOS INTANGIBLES EN UNA INDUSTRIA AEROESPACIAL
}

The knowledge management critical tool in the management of intangible resources in an aerospace industry

\section{Yolanda De La Torre - Martínez}

Doctora en Administración, Universidad Autónoma de Querétaro. Querétaro, México.

yolandadelatorremtz@gmail.com

Norma Maricela Ramos - Salinas

Doctora en Ciencias de Gestión, Universidad Autónoma de Querétaro.

Querétaro, México.nmrs5@hotmail.com

\section{Enrique González - Sosa}

Doctor en Mecánica de Medios Geofísicos y Medio Ambiente, Universidad Autónoma de Querétaro.

Querétaro, México. egs@uaq.mx

\section{Cómo citar / How to cite}

De la Torre-Martinez, A., Ramos-Salinas, N. y González- Sosa, E. (2016). La gestión del conocimiento herramienta decisiva en la gestión de los recursos intangibles en una industria aeroespacial. Revista CEA, 2(3), 31-48.

Recibido: 16 de octubre de 2015

Aceptado: 12 de noviembre de 2015

\section{Resumen}

El presente artículo invita a los investigadores y los prácticos facultativos a interrogarse, cada vez más, sobre los pasos factibles para valorizar, perpetuar, trasladar y crear el conocimiento organizativo. Esta investigación, de tipo mixto descriptivo, fue concebida para revisar los diversos procesos de la gestión del conocimiento en una empresa de la industria aeroespacial, ubicada en la ciudad de Querétaro. Para ello fue realizado un análisis acerca de la eficiencia de sus estrategias en el proceso de la gestión del conocimiento, apoyada por las Tecnologías de Información y Comunicación (TIC). La revisión del fenómeno de la gestión y la conversión del conocimiento consistió en evaluar su incidencia en la industria. La evaluación fue llevada a cabo bajo un caso de estudio en la organización KA (empresa estudiada), apoyándose en encuestas a directivos y empleados en general. Los resultados permitieron identificar las funciones sustantivas y administrativas dentro de la espiral de creación de conocimiento organizacional, en una dimensión epistemológica de creación de conocimiento organizacional. Uno de los principales aciertos de esta investigación fue la identificación del proceso de conversión del conocimiento, mediante la adaptación del conocimiento del personal. Lo que muestra la presencia de un consenso sobre la conversión y apropiación del conocimiento en la industria aeroespacial estudiada. Lo anterior resulta significativo, si se considera que la industria aeroespacial se estableció en Querétaro recientemente, esencialmente porque en la industria nacional existe una necesidad real de capitalizar y de explotar las informaciones nacidas de experiencias pasadas con el fin de mejorar la toma de decisiones y de crear nuevos conocimientos a partir de los recursos intangibles en las organizaciones. 
Palabras Clave: gestión del conocimiento, conversión del conocimiento, industria aeroespacial, estrategias.

\section{Abstract}

This article offers to researchers and practical physicians different questions about feasible steps to promote, perpetuate, move and create organizational knowledge. The current study -of a descriptive mixed type- was carried with the intention to review the processes of knowledge management in an aerospace industry located in Queretaro City. The study was conducted to analyze the efficiency of the strategies in the process of knowledge management through information and communication technologies (ICT). The review of the phenomenon of management and conversion of knowledge included evaluating its presence in the industry field. The study was carried out using a case study of the organization KA (firm studied), based on surveys with the staff. The findings allowed us to highlight the substantive and administrative functions within the spiral fo creation of organizative knowledge, in an epistemological dimension of organizative creation of knowledge. One of the main findings of this study is the identification of the process of conversion of knowledge management through the adaptation of knowledge of the staff. This shows the presence of a consensus about the conversion and appropriation of knowledge in the studied aerospace industry. The latter is especially important if we take into account that the aerospace industry is new in Queretaro, mainly because in the domestic industry there is a real need to use and implement lessons learned in previous experiences in order to improve the decision making process and to create new knowledge from intangible resources in the organizations.

Keywords: knowledge management, conversion of knowledge, aerospace industry, strategies.

\section{INTRODUCCIÓN}

El conocimiento está cada vez más revalorado, como un recurso inmaterial estratégico de las organizaciones, alcanzando una ventaja competitiva decisiva. En este contexto, los progresos de las Tecnologías de la Información y de la Comunicación (TIC) desempeñan un papel fundamental en la recolección y la gestión del conocimiento, que a su vez representa una nueva postura de importancia en numerosos dominios industriales; conviniendo un proceso de creación de valor, a partir de las diferentes fuentes de conocimientos de la organización que pueden estar disponibles bajo formas distintas: en los procedimientos de trabajo, en el espíritu de sus empleados, en las normas, en los documentos internos, en sus bases de datos, entre otros (Bergmann, 2002). Por otro lado, la gestión del conocimiento en sostenimiento emerge como un factor esencial para mejorar los resultados de las empresas, que ampliamente dependen de la calidad del sustento de sus recursos operacionales. Así, esta gestión debe poder permitir preservar y valorizar los conocimientos nacidos del análisis de los acontecimientos o de los hechos pasados con vistas a mejorar los planes de sustento actuales, las estrategias de sostén, el diagnóstico de los equipos, las medidas de prevención que hay que poner en ejecución y/o los procedimientos y las acciones llevadas en el momento de las intervenciones.

\section{LAS RELACIONES DE LA GESTIÓN DEL CONOCIMIENTO EN DESARROLLO INDUSTRIAL}

El conocimiento reside en la persona y es la interpretación de la información (Drucker, 1966; citado por Valhondo 2003). En el lenguaje empresarial de la organización, el conocimiento es un conjunto de información, reglas, interpretaciones y conexiones puestas en un contexto, y de una experiencia que ha logrado la organización, de forma general o personal (Márquez et al. 2007). López, Marulanda y 
Agudelo (2010) sostienen que usar el conocimiento es aplicar lo aprendido en experiencias pasadas para resolver problemas nuevos.

Para Senge (1992), la prosperidad de cualquier organización reside en la habilidad y capacidad de aprender cosas nuevas. Las organizaciones inteligentes explotan la experiencia colectiva, el talento y la capacidad de cada individuo para aprender en conjunto y triunfar. Para Nonaka y Takeuchi (1999) el conocimiento personal de un individuo se transforma en conocimiento organizacional, por medio de metáforas la gente unifica de una manera nueva lo que sabe $y$ empieza a expresar lo que ya conoce.

Para Escorsa (2002) la gestión del conocimiento consiste básicamente en «documentar el conocimiento existente en las empresas y en compartirlo con los trabajadores», citado por Arias et al. (2007), quienes definen a la gestión del conocimiento como la estrategia que convierte los valores intelectuales en valor añadido e incremento de la productividad. La gestión del conocimiento es una estrategia empresarial consciente, cuyo objetivo consiste, a grandes rasgos, en garantizar que el conocimiento adecuado vaya a las personas apropiadas en el momento oportuno y, además, les ayude a compartir y a utilizar la información de tal modo que la empresa sea capaz de mejorar su acción organizativa, así lo sostiene Ordóñez (1999), citado por Martínez (2006).

Gil et al. (2011) considera que el objetivo de la gestión del conocimiento es constituir un stock de conocimientos aplicables a las actividades con objeto de mejorar su eficiencia y, para ello, existen herramientas, procedimientos, reglas $y$ sistemas destinados a captar, tratar, recuperar, presentar y transmitir los datos, informaciones y conocimientos de una organización. Asimismo, Mata y Pesca (2011) concluyen que la gestión del conocimiento es una estrategia gerencial que permite a las organizaciones mantenerse, revitalizarse y permanecer acorde con su misión y visión, según su responsabilidad, social, ética y moral para el país que representa.
Nava, Esis y Naval (2011), citando a Ordóñez (2000), mencionan que los activos intangibles son los que poseen valor sin tener dimensiones físicas, los tienen las personas (empleados, clientes, proveedores) o se obtienen a partir de procesos, sistemas y la cultura organizacional. Estos activos incluyen marcas, conocimiento individual, propiedad intelectual, licencias y conocimiento organizativo.

Smith y Hurtado (2012) consideran el éxito de la gestión del conocimiento como un conjunto dependiente de factores internos y externos a la organización, tales como el diseño organizativo, el cual juega un rol fundamental como elemento facilitador del mismo. Saldarriaga (2013) sostiene que la gestión del conocimiento es una forma de generar productividad y competitividad empresarial, además de ser un aspecto determinante en gran medida en la gestión de la organización. Guillo (2013); citado por Guerrero et al. (2014), establece el proceso de gestión del conocimiento en diferentes etapas: descubrir, capturar, codificar, validar, transferir y convertir la información en accionable del conocimiento.

Armas (2015) encuentra que una adecuada gestión del conocimiento permite impulsar o potenciar el capital intelectual, así como generar ventajas competitivas para la organización; enfatiza que el capital intelectual, por sí solo, no potencia la gestión del conocimiento, ya que únicamente influye en él a medida que la necesidad de incorporar a la organización esa modalidad de gestión sea evidente.

Arellano (2015) define la gestión del conocimiento como un conjunto de procesos y sistemas que permiten que el capital intelectual de una organización se incremente de forma significativa, mediante la gestión de sus capacidades para la solución de problemas y cuyo objetivo es crear ventajas competitivas, así mismo considera que la gestión del conocimiento debe ser utilizada como una estrategia que propicie y facilite la transición de los estados del conocimiento organizacional, 
para conservar el conocimiento de una o varias personas de la organización.

Preguntas de investigación

¿El modelo de gestión del conocimiento de Nonaka y Takeuchi de 1999 facilita el desarrollo de las funciones sustantivas y administrativas en la organización KA, apoyado en el uso eficaz y eficiente de las tecnologías de la información y la comunicación (TI)?

¿Cómo se gestiona el conocimiento y de qué manera influyen las TI en la organización KA?

¿De qué manera la organización KA pone en práctica el uso de la gestión del conocimiento y de las TI?

¿Cuál es la información con la que cuenta el personal de KA de lo que significa la gestión del conocimiento y de las TI en la organización KA?

\section{MARCO TEÓRICO}

\section{La gestión del conocimiento}

Ikujiro Nonaka e Hirotaka Takeuchi en su libro "La organización creadora de conocimiento» (1999), sostienen que el éxito en las compañías japonesas se debe a la capacidad para generar nuevos conocimientos y trasmitirlos a cada uno de los miembros de la institución: empleados de primera línea, ejecutivos y altos directivos, de donde la creación de nuevo conocimiento es producto de una interacción dinámica entre ellos. Para definir la creación de conocimiento organizacional escriben: «Este concepto debe entenderse como la capacidad de una compañía para generar nuevos conocimientos, diseminarlos entre los miembros de la organización y materializarlos en productos, servicios y sistemas». Nonaka y Takeuchi (1999), se centran en la creación de conocimiento, no en el conocimiento en sí. Estos autores dividen en dos partes al conocimiento: conocimiento tácito y conocimiento explícito.
El conocimiento explícito puede expresarse con palabras y números; puede trasmitirse y compartirse fácilmente en forma de datos, fórmulas científicas, procedimientos codificados o principios universales. Este tipo de conocimiento es el que utilizan las empresas occidentales.

Sin embargo, las empresas niponas tienen otra idea con respecto al conocimiento, consideran la expresion con palabras y números como el inicio, pero no como un conocimiento que lleve al éxito a una empresa. Este conocimiento tácito es difícil de expresar con palabras y los individuos, como parte de las organizaciones, lo manejan como una pertenencia, lo que guía a la empresa a un buen desarrollo. El conocimiento tácito se divide en dos dimensiones: la técnica y la actividad cognoscitiva. En la primera, el individuo sabe cómo realizar una tarea o trabajo; la segunda, incluye esquemas, modelos mentales, creencias y percepciones. Esto último se va adquiriendo por las experiencias vividas; es difícil comunicarlo a los demás y solamente a través de la práctica podría transformarse en explícito. «El conocimiento más profundo no se puede trasmitir o enseñar a otros» (Nonaka y Takeuchi 1999). Enseguida se presenta en la Tabla 1, un análisis comparativo de algunas teorías referentes a la gestión del conocimiento.

La creación de conocimiento organizacional es una interacción continua de conocimiento tácito y conocimiento explícito. El personal se instala con conocimiento tácito para que sea entendida por los mismos miembros del grupo, estos deben tener riqueza del lenguaje figurativo e imaginación de los líderes y se puede concluir que la exteriorización es la clave de la creación del conocimiento en las cuatro formas de conversión de este.

Para la creación de conocimiento organizacional debe existir una interacción continua de conocimiento tácito y conocimiento explícito, y para ello se requiere llevar a cabo la conversión de conocimiento. Primero, debe ser la socialización o transformación de conocimiento tácito-tácito y produce un conocimiento 
armonizado; segundo, después la exteriorización o transformación de tácito a explícito para incluir metáforas y analogías que faciliten la comunicación, entonces se obtiene un conocimiento conceptual; tercero, se utiliza la combinación o transformación de explícito a explícito y así se logra distribuir el conocimiento a todas las secciones de la organización y se obtiene un conocimiento sistemático; cuarto, la interiorización o transformación de explícito a tácito da un conocimiento operacional, como se muestra en la Figura 1.

Tabla1. Algunas teorías referentes a la gestión del conocimiento

Table 1. Some theories concerning the management of the knowledge

\begin{tabular}{|c|c|}
\hline Autores / Año & Aporte \\
\hline Peter F. Drucker/ 1966 & $\begin{array}{l}\text { El conocimiento reside en la persona y es la interpretación de la } \\
\text { información. }\end{array}$ \\
\hline Michael Polanyi /1967 & $\begin{array}{l}\text { El conocimiento verdadero no es posible explicarlo por un conjunto de } \\
\text { reglas articuladas; es público, pero también es personal y emocional. } \\
\text { Existe un conocimiento que subyace al conocimiento explícito que es } \\
\text { fundamental: se trata del conocimiento tácito. }\end{array}$ \\
\hline Peter Senge /1992 & $\begin{array}{l}\text { La prosperidad de cualquier organización reside en la habilidad y } \\
\text { capacidad de aprender cosas nuevas. Las organizaciones inteligentes } \\
\text { explotan la experiencia colectiva, el talento y la capacidad de cada } \\
\text { individuo para aprender en conjunto y triunfar. }\end{array}$ \\
\hline Nonaka y Takeuchi /1994 & $\begin{array}{l}\text { El conocimiento personal de un individuo se transforma en } \\
\text { conocimiento organizacional. } \\
\text { Por medio de metáforas la gente unifica de una manera nueva lo que } \\
\text { sabe, y empieza a expresar lo que ya conoce, pero aún no puede } \\
\text { explicar. } \\
\text { La analogía es una comparación entre dos ideas y objetos. } \\
\text { La creación del conocimiento se basa en el conocimiento tácito y el } \\
\text { conocimiento explícito, y hacen cuatro conversiones de conocimiento: } \\
\text { tácito a tácito (socialización), tácito a explícito exteriorización), explícito } \\
\text { a explícito (combinación) y explícito a tácito (interiorización). }\end{array}$ \\
\hline Chrys Argyris / 2002 & $\begin{array}{l}\text { El conocimiento solo es posible a través del aprendizaje. El } \\
\text { conocimiento de un solo circuito es para lo rutinario (puede ser } \\
\text { relevante para la supervivencia de largo plazo) y lo repetitivo, el } \\
\text { conocimiento a doble circuito es para aspectos complejos no } \\
\text { programables. El conocimiento solo es posible a través del aprendizaje. }\end{array}$ \\
\hline
\end{tabular}

Fuente: Nonaka y Takeuchi 1999, Argyris 2002, Valhondo 2003, Senge 2012. 


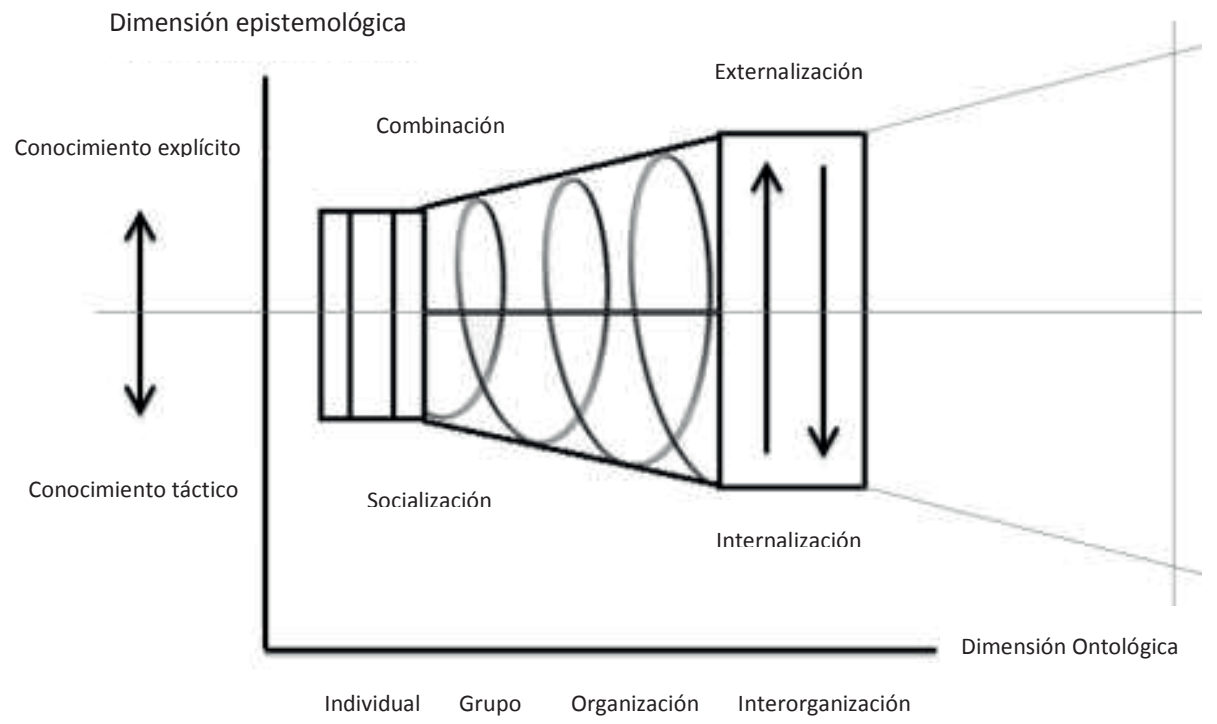

Figura 1. Espiral de creación de conocimiento organizacional

Figure 1. Spiral of organizational knowledge creation

Fuente: Nonaka y Takeuchi (1999).

\section{Las Tecnologías de la Información y Comunicación (TIC) en las organizaciones}

Laud y Thies (1997); Gándara et al. (2007) afirman que las TIC fomentan el cambio y también son capaces de proporcionar sistemas de control y de planeación integral, ya que proveen de herramientas necesarias para promover la toma de decisiones en las diferentes áreas de la organización. Sin embargo, en la actualidad algunas organizaciones solo emplean las TIC en labores rutinarias dentro de la administración y no para prácticas realmente de valor dentro de la empresa; deberían aplicarse para crear una verdadera acción que transforme a la empresa, incorporando audacia que se logra con ese recurso estratégico.

Macau (2004) expone que la realización de las actividades de producción de valor y sus enlaces son los que el directivo debe gestionar, y no puede hacerlo correctamente sin tener en cuenta el componente físico y el componente informativo de dichos enlaces y actividades. De acuerdo con Sheel y Rivera (2009) las TIC son condiciones necesarias para determinar la competitividad de las organizaciones, en conjunto con las condiciones propicias del entorno. Aseguran que las tecnologías medulares más utilizadas actualmente por las empresas son: logística, manufactura y el Enterprise Resource Planning (ERP, software para planeación de recursos), para funciones administrativas; estos últimos son necesarios, pero no suficientes para producir altos rendimientos.

Scheel y Rivera (2009) plantean que, en las organizaciones latinoamericanas, las TIC se utilizan principalmente en actividades administrativas rutinarias y no en apalancar ventajas competitivas, y su uso no está alineado con el desarrollo de competencias medulares.

Gándara et al. (2007) sustentan que la gestión del conocimiento y las TIC son un recurso y habilidad capaces de crear valor en las empresas que las utilicen. Cuando estas invierten en las tecnologías de información y comunicación deben esforzarse para alcanzar mejores resultados, aunque implique la aplicación de recursos financieros y humanos. Así mismo, las TIC no deben considerarse agentes aislados, son facilitadores de ventajas competitivas y se están convirtiendo en un 
factor de éxito, por lo que González, Soto, Molina, Trigo y Varajao (2010) recomiendan que los directivos y los mandos medios de una organización vean a las TI y a la gestión electrónica como fuente de información global e integral, y también a los agentes con los que se interactúa principalmente, que son los proveedores, clientes y empleados.

Scheely y Rivera (2009) observan que en las empresas latinoamericanas el uso de las TIC no están orientadas hacia la competitividad, que lo urgente se antepone a lo importante, volviéndose común el temor al cambio, al riesgo y la desconfianza sobre la práctica de compartir información entre diferentes áreas de las empresas, actitud que impide la integración de los procesos y las estrategias. Así mismo observan la falta de capacitación y entrenamiento del personal.

En consecuencia, es importante hacer énfasis en las variables asociadas con las TIC, así como las relacionadas con la gestión del conocimiento, las cuales son abordadas en el caso de la empresa KA, que forma parte del clúster aeroespacial, situado en el corredor industrial del centro de México, en los estados de Querétaro, Guanajuato, Aguas Calientes y San Luis Potosí.

\section{LA GESTIÓN DEL CONOCIMIENTO EL CASO DE LA INDUSTRIA AEREOESPACIAL EN MÉXICO}

Los procesos de la gestión del conocimiento en una empresa de la industria aeroespacial ubicada en la ciudad de Querétaro, estado que en la última década ha mostrado un crecimiento importante y en plena consolidación. Condición que conlleva a un análisis para identificar los elementos de éxito de este sector en un país donde la industria aeroespacial es relativamente nueva en comparación con la industria automotriz, con una historia de más de 60 años en México.
Esta investigación de tipo mixta descriptiva y concebida para revisar que el proceso de gestión del conocimiento y las TIC influyen positivamente en la organización KA. Pretende probar si la organización KA hace uso de las prácticas de la gestión del conocimiento y las TIC, así como si el personal de la empresa cuenta con información del proceso de las mismas.

La estrategia del enfoque metodológico en este trabajo es mixta, no experimental, transversal, dado que el contacto con el individuo estudiado fue una única vez. Se inicia con el enfoque cualitativo, a través de un estudio de caso, donde se realizaron entrevistas abiertas, seguidas de observación, dando como resultado la validación de un cuestionario para aplicarse en la empresa. Esto corrobora el empleo mixto de la metodología, conocida también por triangulación del investigador.

Para el enfoque cuantitativo que implica la recolección y el análisis de datos cuantitativos y su integración y discusión conjunta para entender el fenómeno estudiado. Se reconoce al individuo como constructor de la realidad, y a la empresa KA y las TIC como el objeto de la investigación. Se busca encontrar la respuesta a la pregunta central: ¿El modelo de gestión del conocimiento de Nonaka y Takeuchi de 1999 facilita el desarrollo de las funciones sustantivas y administrativas en la organización KA, apoyado en el uso eficaz y eficiente de las tecnologías de la información y la comunicación $(\mathrm{TIC})$ ?

El cuestionario empleado se encuentra dividido en dos bloques con un total de 52 preguntas generales, utilizando la escala de Likert con rangos de 5 puntos, donde 1 equivale a "totalmente en desacuerdo», y el 5 equivale a «totalmente de acuerdo». Finalmente se realizó un análisis descriptivo de los resultados de la investigación cuantitativa.

Los conceptos de investigación son: la gestión del conocimiento y las TIC. Tomando el modelo de investigación y metodología de la tesis 
sigmaplot, algunos de estos se presentan enseguida.

La representatividad de los datos fueron validados con la prueba Alfa de Cronbach, cuyo valor es de 0.946, lo cual califica como confiable y proporciona la validez requerida al instrumento de medición. En la Figura 2, son mostrados los resultados.

(socialización,
exteriorización, combinación interiorización), madurez de la gestión del conocimiento, no implementación.

- Tecnologías de la Información y la Comunicación (TIC): fuente de ventaja, actitudes, inversión, uso.

La población objeto de este estudio son los ochenta empleados en KA. La validez del tamaño de la muestra es el resultado de la aplicación del cálculo del tamaño de la muestra, que es de 68 encuestas, con un nivel de confianza del $99 \%$, considerando un margen de error del 7\%. El periodo de recolección de datos fue entre los meses de abril a julio de 2013. Entre las principales limitantes durante el proceso fue la gran cantidad de trabajo $y$ diferencia de horarios de labores, por lo que hubo personas que no respondieron las encuestas, en total se obtuvo $85 \%$ de las encuestas objetivo equivalente al $100 \%$ de la prueba estadística (68 encuestas).

La revisión documental fue realizada en diferentes fases, comprendidas entre los meses de mayo a septiembre del 2013. Las 68 encuestas aplicadas al personal de KA cubre varios niveles de la organización, como: operadores, técnicos, administrativos (mandos medios), jefes de departamento y gerentes. El análisis estadístico fue llevado a cabo con el software SPSS (programa computacional para la obtención de estadísticas y para dar validez a los datos por medio de análisis) y al software Excel, para el procesamiento de la información se utilizaron gráficos en el software Excel (estadística descriptiva), Igualmente el SPSS para obtener el Alfa de Cronbach e histogramas (estadística inferencial) a través del software

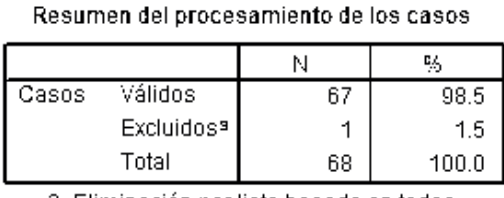

a. Eliminación por lista basada en todas las variables del procedimiento.

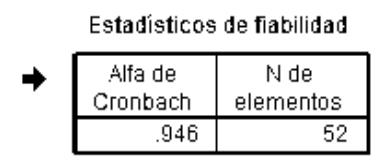

Figura 2. Alfa de Cronbach

Figure 2. Cronbach's alpha

Fuente: elaboración propia con SPSS

En cuanto al análisis descriptivo, el comportamiento de las variables en la organización KA es presentado en la Figura 3. Son mostradas las actividades que explican la interiorización (conocimiento explícito a tácito) de la empresa y muestran la oportunidad de mejorar en la interiorización.

Contextualmente, dichas actividades de interiorización concebidas como "aprender al hacer»; reflejan una aceptación de los empleados, sin importar el rango. En otras palabras, comparten, aceptan y permiten la simulación $y / o$ experimentación con procesos y/o productos a partir de manuales, best practices, historias orales, documentos, entre otros, con un porcentaje entre el $44 \%$ y $31 \%$ que manifiesta estar más o menos de acuerdo. Particularmente, el personal administrativo opina, con un porcentaje del $18 \%$, estar no tan de acuerdo, y un porcentaje de $9 \%$ está en desacuerdo. Estos datos nos indican que el personal practica de manera aislada el «aprender al hacer». 


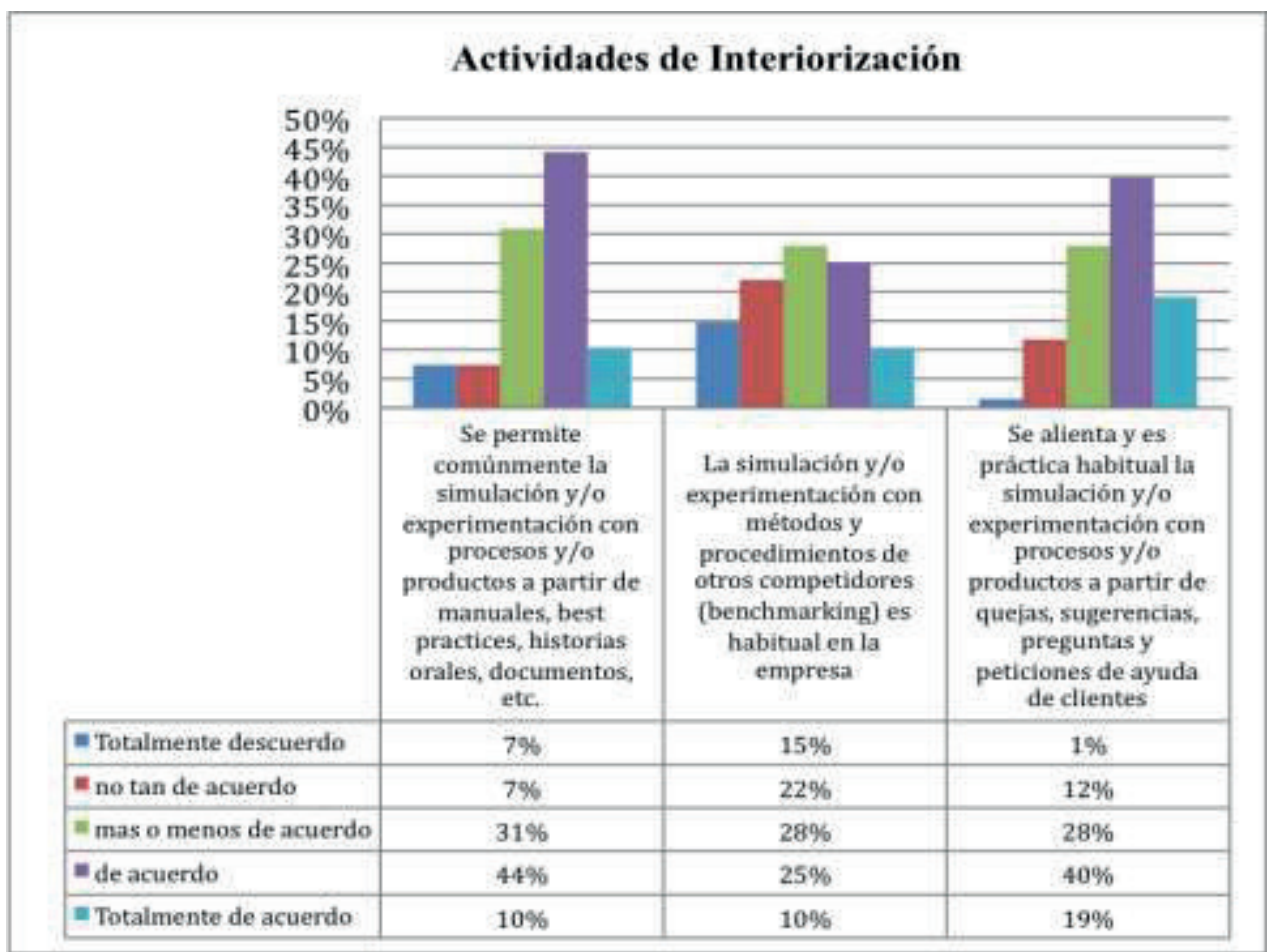

Figura 3. Actividades de interiorización (conocimiento explícito a tácito) (n=68)

Figure 3. Activities of internalization (tacit to explicit knowledge) $(n=68)$

Fuente: elaboración propia

El modelo lineal mostrado en la Figura 4, que relaciona las actitudes versus act. social, arrojó una alta correlación, con un nivel de confianza del 95\% de predicción. Puede observarse que las variables de las actitudes de gestión del conocimiento (variable dependiente) está correlacionada en forma positiva y ascendente, con las actividades de socialización logrado a través del trabajo. Las diferencias en los valores medios entre los grupos estudiados son mayores que lo que se esperaría por oportunidad; existe una diferencia estadísticamente significativa $(P=<0,001)$. La prueba realizada tiene un alfa $=0.050: 1.000$ Medias cuadradas esperadas.

Puede interpretarse que los ítems de gestión del conocimiento versus los ítems de las actividades de socialización en la organización KA están directamente relacionados con la gestión del conocimiento, debido a que estas les proporcionan una identidad y valor como individuos, como parte de la empresa. 


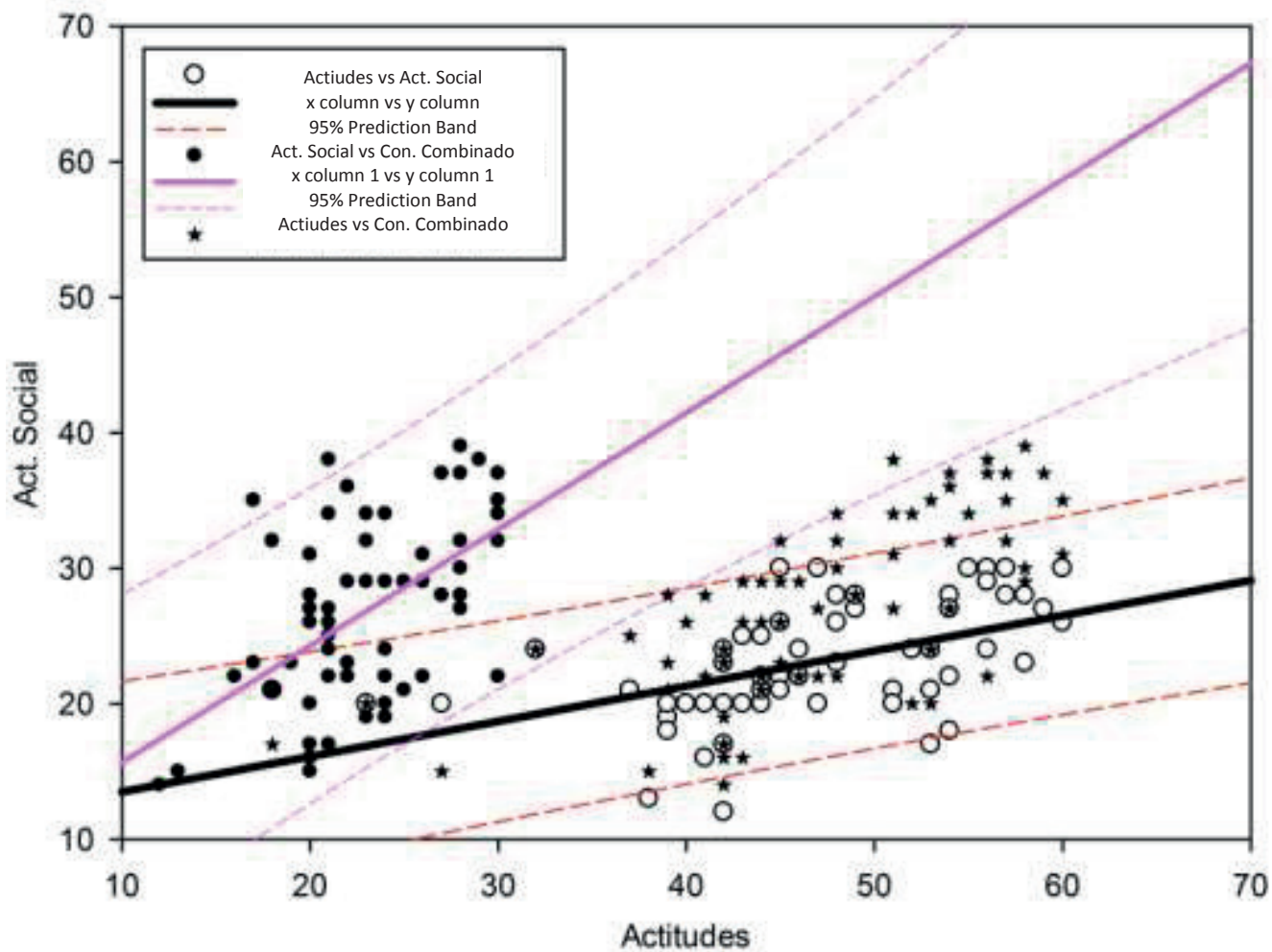

Figura 4. La prueba de regresión lineal

Figure 4. Linear regression test

Fuente: elaboración propia con SigmaPlot, ver 12.5

Los resultados de la correlación lineal y la prueba de Alfa de Cronbach, así como el análisis de los resultados, nos permite postular los aspectos siguientes:

La acertada decisión tomada al inicio de operaciones la empresa KA (empresa metalmecánica del giro aeroespacial), que seleccionó a un grupo de personas provenientes de la empresa TA (empresa metalmecánica del giro automotriz de la cual KA es filial) para enviarlas a Canadá a la empresa MD (empresa metalmecánica del giro aeroespacial) con el objeto de estudiar las técnicas que esta empresa (MD) aprovecha para llevar a cabo la elaboración de sus productos, con la finalidad de que piezas similares se elaborarán en KA. Con la intención de que las experiencias adquiridas fueran aplicadas en KA, mediante metáforas y analogías, para construir elementos semejantes utilizando los mismos principios, teniendo en cuenta las limitaciones que la maquinaria y con un personal más modesto para trabajar KA, se muestra que el "aprender al hacer» adquirido y una gestión pertinente del conocimiento son parte de su éxito.

En forma sucinta podemos destacar cómo la gestión del conocimiento en la empresa KA se lleva a cabo, y distingue las cuatro formas de transformación del conocimiento:

En primer término, se adopta el conocimiento tácito transformándolo a tácito, por medio de la socialización, donde se comparten experiencias en modelos mentales y habilidades técnicas. El entrenamiento informal de los empleados se manifiesta cuando se apropian de la técnica; el personal de nuevo ingreso consulta y pregunta a otros que tienen conocimiento, el cual se transfiere de persona a persona, sin un plan de trabajo definido. Al personal de nuevo ingreso es apoyado por una persona experimentada para facilitar su aprendizaje dentro de la 
empresa, mediante la observación, aprende y consulta lo que necesita saber (el saber hacer). El personal de administración de proyectos también apoya la gestión del conocimiento, ya que tiene el contacto directo con el cliente, realiza juntas semanales para exponer al resto de la empresa las necesidades e inquietudes de ellos.

La segunda forma de conversión es la exteriorización, la cual establece la conversión de conocimiento tácito a explícito, es decir, enuncia el conocimiento tácito en forma de conceptos explícitos; esto se da en la empresa al adoptar analogías, conceptos y modelos promoviendo la reflexión y la interacción en formas colectivas entre el personal de cada área. La analogía que el personal hace con respecto a lo aprendido en previos empleos se establece con sus respectivas adaptaciones, enfocándolo a modelos que la empresa tiene como resultado final.

La tercera forma de conversión de conocimiento es de explícito a explícito, si se presenta la necesidad implementa simulaciones con procesos de manufactura con sus respectivas pruebas. La interacción es establecida cuando un cliente lo solicita o bien cuando se implementa una pieza nueva. El benchmarking no es habitual.

La cuarta forma de conversión es la interiorización de conocimiento explícito a tácito, que está relacionada con el aprendiendohaciendo. La empresa KA lo formaliza a través de la verbalización, diagramas, documentos y manuales. Interiorizan lo experimentado enriqueciendo su conocimiento tácito, además, se experimenta la vivencia de otras personas. El diálogo con los clientes se realiza través del ingeniero de proyectos $\mathrm{y} / \mathrm{o}$ del planeador maestro; a través de ellos se obtienen los comentarios y se transmiten a la organización o viceversa. Otra forma, menos común, se facilita por medio de calidad cuando hay rechazos de piezas y estas vienen acompañadas de una queja y de la petición para que se explique por qué se produjo, y a partir de ahí se ordenan las acciones correctivas.

El inicio de operaciones en KA fue identificar los procesos que deberían realizarse y el esquema para llevarlos a cabo; el personal realizó un benchmarking, para lo cual tuvo un entrenamiento apoyándose en el sistema de calidad de la industria aeroespacial: AS9100, esencial para diseñar e implementar los procesos de la empresa.

Una vez adquiridos los conocimientos del sistema fue reconocida la necesidad de información, distribución del trabajo por áreas, contratación de personal externo de consultoría para entender a la industria y el tipo de piezas; a través de las actividades crearon procedimientos y manuales, e inició la simulación de los procesos de manufactura; el proceso de calidad de la organización fue configurado a partir de la herramienta APQP (Planeación Avanzada de la Calidad del Producto), utilizada por la industria automotriz.

Para que el sistema de calidad fuera aprobado y llevado a la práctica, recolectaron la información fundamental de cada proceso, así como los procedimientos, tales como:

- Una pieza manufacturada transita por la organización con la información detallada, en caso de carecer de ella, no puede avanzar a la siguiente operación.

- Los datos son analizados; una vez validados y correctos pasan a la siguiente operación, en caso de requerir una mejoría, en la información o en los procesos, esta se realiza para continuar en la cadena de producción y continúa avanzando.

- Es difundida y se comparte la información con la organización y se procede a su uso en la cadena de producción.

La funcionalidad y aprobación del sistema de calidad, de manera recurrente, funciona de la siguiente manera: 
- Recolección de la información y documentación de las etapas para cada uno de los procesos.

- Enseguida se detalla la información de la pieza por manufacturar para seguir el proceso, una vez validada la información del proceso.

- Validada la información, de manera detallada, por los participantes de cada proceso, pasa a la siguiente fase.

- Se verifica si se requiere de mejoras en procesos e información.

- Finalmente se comparte la información a la organización y se procede a su uso.

La forma de «aprender haciendo» ancla la interiorización (conversión de conocimiento explícito a tácito) para crear el conocimiento operacional y de esta forma lograrse el impulso de la espiral de creación de conocimiento organizacional. Podemos apreciar que esta es la manera como KA ha provocado el sentido de pertenencia a sus trabajadores, enviándolos en primera instancia a la capacitación en una empresa en Canadá para establecer analogías de los procesos productivos, posteriormente formándolos en cursos de capacitación para obtener la certificación AS9100, para la adquisición del concepto de trabajo en equipo.

De acuerdo con Ramos, N. M. y González, E. (2013) quienes señalan que «el poder de la tecnología acelera la alineación de la estrategia de las empresas y la estrategia de los sistemas de información», los resultados obtenidos confirman esta hipótesis de la investigación. Igualmente, cabe señalar que la empresa estudiada actualiza constantemente a su personal, al mismo ritmo que emergen nuevas herramientas, a fin de mantenerse en una posición de vanguardia y de productividad competitiva.

\section{DISCUSIÓN}

A la luz de las propuestas teóricas revisadas sobre la gestión del conocimiento, se describe la principal información derivada del análisis del caso, estudiado en forma general en la empresa KA Industria Aeroespacial, la cual por cuestiones de confidencialidad fue nombrada así a lo largo del texto, misma que se encuentra ubicada en una zona industrial en Querétaro, Qro. México.

\section{Antecedentes de los empleados de KA}

El personal de la empresa KA, la muestra del análisis, son trabajadores que disponen de experiencia previa en la industria automotriz y en la actualidad se desempeña en el sector de la industria aeroespacial. Un porcentaje alto (90\%) de la muestra seleccionada para el estudio proviene de empresas manufactureras de la industria automotriz, con maquinaria similar o igual a la que se utiliza en KA para la industria aeroespacial. Casualmente, los principios básicos de transformación son similares y los parámetros de medición idénticos, la diferencia más importante radica en el grado de especialización del personal de todos los niveles (el dominio mínimo de los procesos del puesto que se esté desempeñando). El mayor obstáculo radica en la falta de conocimiento especializado; el cual se va adquiriendo por el estudio, las vivencias propias del trabajo realizado y por las experiencias compartidas.

\section{La comunicación en la empresa KA}

La empresa KA forma parte de asociaciones donde están involucrados los competidores, a través de los mismos se establece la conversación e interacción con respeto y restricción de información. El gerente comercial y el gerente general establecen foros adecuados para la comunicación, donde se establecen entrenamientos formales con cursos dentro y fuera de la empresa, dirigida por expertos internos y externos, enfocados mayormente a la manufactura y al área operativa. El acceso a la información especializada es mediante revistas, 
manuales, libros, foros, cursos, la cual no es accesible a todos los niveles.

Las estrategias de la empresa son diseñadas con base en un mecanismo conocido como balance score card; de manera externa una institución está encargada de facilitar la información, análisis del mercado y de la industria que lo demande. La información es restringida al público en general y se presenta en interno por medio de reuniones, tableros informativos, pizarrones, share point o correos electrónicos. Una vez realizadas las observaciones, se sugiere diseñar una estrategia que permita a todo el personal tener el acceso a la información.

\section{Herramientas de conocimiento en KA}

La creación de conocimiento organizacional es una interacción continua de conocimiento tácito y explícito. La espiral de conocimiento circula en sus dos dimensiones como son la ontológica y la epistemológica completando con esto la formación del individuo para beneficio de la empresa y del individuo mismo.

El uso de la Planeación Avanzada de la Calidad del Producto (APQP), metodología empleada en la industria automotriz para asegurar la producción de una pieza con la calidad requerida bajo normas y costo, en un tiempo determinado. Particularmente, el conocimiento tácito considerando en el análisis del caso contempla la experiencia previa de los trabajadores y considera la adquirida para transformarla en explícita, lo cual condujo a la empresa KA a diseminarla entre sus empleados. Cumpliendo así con la exteriorización, que es el conocimiento tácito que pasa a ser explícito.

El uso del manufacturing package es un conocimiento explícito que se transmite a todo aquel que participa en toda la cadena productiva de KA. La conversión de este conocimiento es de explícito a explícito es coincidente con la propuesta de Nonaka y Takeuchi, considerando que la combinación es un proceso de sistematización de conceptos, con el que se genera un sistema de conocimiento.

La empresa KA cuenta con tres niveles de motivación de acuerdo al puesto:

1. Para el equipo champion (gerentes y jefes), el objetivo es preparar un negocio rentable, bajo este esquema se han desarrollado sistemas bajo lo cual se califica su desempeño.

2. Para los mandos medios, el propósito es cumplir los objetivos del equipo del cual son parte y, con base en este criterio, es calificada su ejecución.

3. Para el nivel operativo, se maneja un bono de productividad de acuerdo con la eficiencia, niveles de desecho, entregas a tiempo, registros, accidentes; se inicia con un sueldo base y si se cumple con todo lo anterior se hace acreedor a un incentivo económico.

En general, se califica a todo el personal de acuerdo con el desempeño del equipo en que operan. Hasta el momento se ha identificado una falta comunicación, más allá de los resultados de producción, considerando que no todo el personal está enterado sobre cómo se administra la empresa y si algunos procedimientos no han sido establecidos; el personal tiene una idea (algunos más clara que otros) de cuáles son las funciones de cada puesto. Se observa que aún no se cuenta con directorios de expertos en la organización; la parte gerencial considera que han sido bien definidas las áreas.

El conocimiento se comparte por medio de una plataforma de tecnologías de información que guardan los documentos de procesos y procedimientos más importantes de la organización. El flujo de información comienza con los jefes; se tienen los diagramas de flujo de datos, los cuales están en la plataforma de Tecnologías de Información y Comunicación (TIC), los empleados los conocen solo si se necesita o se consulta de manera individual. 
El equipo champion (jefes y gerentes) están trabajando en los manuales del proceso, tienen un avance del $80 \%$, a la fecha en que se levantaron datos en esta investigación. Existe un nuevo esquema de trabajo por grupos liderados por el equipo champion, se le denomina $A 3, y$ está integrado por diferentes grupos y quienes los dirigen; el A3 está formado por diferentes áreas, dividido por procesos operativos; su nombre hace referencia a la hoja de ese tamaño, donde se plasman gráficas de indicadores, resultados y actividades.

Los grupos son interdisciplinarios, conformados por personas de diferentes áreas, y cada una se comunica con su equipo o directamente con su jefe. El seguimiento es diario y los equipos exponen avances una vez a la semana; en esta actividad los jefes están presente y es a ellos a quienes se les comunica las actividades realizadas o pendientes, y son quienes, a la vez, lo comunican al resto de los equipos.

Los instrumentos que facilitan el flujo de trabajo son: A3, tableros informativos, SAP, pizarrones y share point. La organización fomenta que el conocimiento se genere y se transmita. Los jefes aseguran que hay que comunicar todo; se observó que no existe un proceso o diagrama de cómo fluye la comunicación.

El sistema de calidad AS9100 obliga a que toda actividad referente a la manufactura de una pieza debe quedar documentada, en este caso se realiza en la plataforma virtual de TIC con el objetivo de que toda la organización tenga acceso. Asimismo, está información debe transmitirse a los operarios e indicar cómo hacer los componentes con un instructivo realizado previamente por el ingeniero de manufactura. Los operarios aprenden observando de éste último, quien deja instrucciones claras para que se repita la operación.

La medición de resultados referente a la gestión del conocimiento se da por medio de la herramienta $A 3$; existen los indicadores de cada equipo que van ligados a las ventas $y$ productividad.

La Conversión del Conocimiento en KA, se puede comparar con la espiral de creación de conocimiento organizacional de Nonaka y Takeuchi (1999). En la dimensión ontológica, el conocimiento individual se convirtió en conocimiento de grupo, y de ahí continuó la secuencia de trasladarlo al organizacional y luego al interorganizacional. De lo anterior, como hallazgo en esta investigación se deriva la definición de adaptación del conocimiento: "Acomodar el conocimiento tácito que el individuo trae de sus experiencias vividas a las condiciones de la organización» (De la Torre, 2015).

Valhondo señala en su libro sobre la Gestión del conocimiento, del mito a la realidad (2003), la interacción que entre la información y las personas produce el conocimiento, ya que a su vez produce más conocimiento para interacciones adicionales. Todos los empleados en KA son trabajadores del conocimiento y este es el auténtico valor de la compañía. Las TIC son parte de la gestión del conocimiento cuando esta ya se tiene dominada.

\section{CONCLUSIONES}

La contribución principal de esta investigación se encuentra en las etapas de práctica, con el fin de estructurar una base de conocimientos específicos que se relacionan con la información que identifica las experiencias acumuladas, para generar una base de conocimientos extraídos bajo la forma de conocimientos más genéricos, considerando la generalización del resultado de cuantiosas experiencias que tienen en común un soporte en la dimensión ontológica.

La actualización o el mantenimiento de las experiencias es un punto decisivo que debería ser abordado y estudiado con el fin de estimar la periodicidad del proceso de extracción de conocimientos en una empresa, la manera con la cual los conocimientos serán actualizados 
(Tseng y al., 2007), y cómo los nuevos conocimientos extraídos serán indizados para la base existente de reglas con el fin de preservar, actualizar y mejorar los conocimientos estratégicos de la organización. Para esto, la base de datos fuente, y su frecuencia de actualización, deben ser analizadas y evaluadas. Estos análisis deben conducir a una estrategia para extraer e indizar nuevos conocimientos $y$ suprimir o modificar los conocimientos ya existentes.

Un tema de investigación importante, colocado en vanguardia a nivel nacional e internacional, es sin duda la empresa basada en el conocimiento. Este trabajo se inscribe en este tema y contribuye a la literatura al realizar un análisis sobre la gestión del conocimiento que se ha ocupado del desarrollo de enfoques y herramientas para ámbitos organizacionales. Bajo esta perspectiva, Ma \& Yu (2010) señalan que los temas principales de investigación en el campo han sido las bases de la gestión del conocimiento, empresa basada en el conocimiento e innovación, aprendizaje organizacional y la estrategia de gestión del conocimiento, en un estudio realizado en el periodo 2007-2008.

La gestión del conocimiento, con frecuencia genera soportes en las TIC con la premisa de que la existencia de la herramienta va a favorecer los procesos de creación de conocimiento. Sin embargo, estos fragmentos de conocimientos -o conocimientos específicos (experiencias vividas)- situados en un contexto particular necesitan un esfuerzo para ser generalizadas. En este contexto, Grundstein (2004), establece que la importancia de la gestión del conocimiento en las empresas induce a una diversidad de herramientas $y / 0$ pasos para reparar, preservar, valorizar y actualizar los conocimientos estratégicos de una organización.

Con base en la pregunta de investigación planteada en este artículo: ¿El modelo de gestión del conocimiento de Nonaka y Takeuchi de 1999 facilita el desarrollo de las funciones sustantivas y administrativas en la organización $K A$, apoyado en el uso eficaz y eficiente de las Tecnologías de la Información y las Comunicaciones (TIC)? Se puede dar una respuesta afirmativa, pues tras haber analizado la gestión del conocimiento de dicha empresa metalmecánica del giro aeroespacial, se evidencia que existe un proceso de conversión del conocimiento (empresa), que comenzó por la adaptación del conocimiento del personal procedente de TA.

Por lo tanto, la gestión del conocimiento es un gran reto que las empresas del siglo XXI afrontan para aprovechar el acervo organizacional; la forma de analizarlas y medir sus beneficios requiere nuevos marcos para enfrentar a las empresas del futuro. Esperamos que esta investigación sirva como plataforma para ampliar el estudio de las prácticas, además de la información que genera la gestión del conocimiento en una organización, ya que tiene como base la política de dirección de la persona para facilitar que el conocimiento de cada una de ellas se convierta en una habilidad para capitalizar sus aportaciones, a partir de su conocimiento, con esto se puede decir que para hacer útil ese conocimiento es imprescindible una actitud abierta al aprendizaje.

La exhortación de esta investigación es la de trascender el aprender haciendo, y el aprender razonando. Es decir, fusionar las corrientes de la GC occidentales y orientales de manera sistémica. El conocimiento tácito, significa tomar en cuenta el conocimiento grupal más de lo que se ha venido haciendo en la actualidad, tomando en cuenta al individuo, enfocándose con esto a la parte más formal y sistemática del conocimiento.

Es fundamental señalar que el tema abordado en el presente artículo es relevante. Se trata de un fenómeno que ha sido abordado desde distintas perspectivas, por tanto, presenta múltiples oportunidades para futuras investigaciones. Desde una perspectiva administrativa, por ejemplo, se podría estudiar cómo solucionar el problema de escasez de 
técnicos especializados en la industria aeroespacial en Querétaro.

Es de suma importancia destacar que la formalización de los conocimientos es un mecanismo indispensable para valorizar $y$ facilitar la reutilización futura de los conocimientos acumulados, como sucede en la empresa KA (empresa metalmecánica del giro aeroespacial) o en otras industrias. En esta investigación se propone como un medio para compartir los conocimientos, favoreciendo su comprensión por medio de representaciones explícitas (Rakoto, 2004) y por un tratamiento más automatizado por la información.

Por otro lado, las cantidades de datos almacenadas en las empresas aumentan de manera exponencial y su explotación es de gran interés. Al referirse al reto de negociar grandes volúmenes de datos, no cabe duda de que las empresas van a explotarlos más en el futuro, Big Data (Chen y Zhang, 2014), para crear ventajas competitivas. Bajo este argumento, la gestión del conocimiento se ha convirtiendo en un recurso que tiene la capacidad de crear valor para aquellas empresas que lo utilicen en todas sus extensiones.

\section{REFERENCIAS}

Arceo Moheno, G. (2009). El Impacto de la gestión del conocimiento y las tecnologías de información en la innovación: un estudio en las PYME del sector agroalimentario de Cataluña. (Tesis inédita de doctorado). Universidad Politécnica de Cataluña.

Arellano Morales, M F; (2015). Gestión del conocimiento como estrategia para lograr ventajas competitivas en las organizaciones petroleras. Orbis. Revista Científica Ciencias Humanas, pp. 31-47. Recuperado de http://sociales.redalyc.org/articulo.oa?id $=70932870004$
Argyris, C. (2001). Sobre el aprendizaje organizacional, México: Oxford University Press.

Arias, L., Portilla, L.M, Villa, C.L. (2007). Gestión del conocimiento: El triunfo de los intangibles. Scientia Et Technica. 13(035). pp. 351-355.

Armas, Y. (2015). La gestión del conocimiento, potenciado por el capital intellectual. Revista Contribuciones a la Economía.

Bergmann, R. (2002), Experience management: foundations, development methodology, and internet-based applications. Lecture Notes in Computer Science, Springer-Verlag Berlin, Heidelberg. 2432

Castaño, J. C., Arias, L., Lanzas, Á. M. (2006) Un cuadro de mando integral para la gestión del conocimiento. Scientia Et Technica. 12(31). pp 153-158.

Chen, Y. J. (2010). Development of a method for ontology-based empirical knowledge representation and reasoning. Decision Support Systems 50(1), 1-20.

Chen, C. L. P. \& Zhang, C. Y. (2014), Dataintensive applications, challenges, techniques and technologies: A survey on big data, Information Sciences 275, 314347.

Escorsa Castells, P. (2002). De la vigilancia tecnológica a la inteligencia competitiva en las empresas. Conferencia ignaural de los estudios de información y documentación de la UOQ del segundo semestre del curso 2001 $-2002$

Gairín Sallán, J.; Rodríguez Gómez, D.; Armengól Asparó, C.; (2007) Funciones y formación del moderador: gestor de redes de gestión del conocimiento, Teoría de la educación. Educación y cultura en la sociedad de la informació , 8(2), pp 55-68 
Gándara, J., Mathison, L., Primera, C, García, L. (2007) Efectos de las TIC en las Nuevas Estructuras Organizativas de la Gerencia Vertical a la Empresa Horisontal. Revista Negotium, 3 (8) pp 4-29

Gil-Montelongo, M. D.; López-Orozco, G.; Molina-García, C.; Bolio-Yris, C. A. (2011) La gestión de la información como base de una iniciativa de gestión del conocimiento. Ingeniería Industrial. (32)3, pp. 231-237.

González-Gallegos, N., Soto-Acosta, P., Trigo, A., Molina-Castillo, F, Varajao, J., (2010) El papel de las TIC en el rendimiento de las cadenas de suministro: el caso de las grandes empresas de España y Portugal Universia Business Review (28) pp. 102-115

Grundstein, M., \& Rosenthal-Sabroux, C. (2004). GAMETH $^{\circledR}$, A Decision Support Approach to Identify and Locate Potential Crucial Knowledge. In D. Remenyi (Ed.), Proceedings 5th European Conference on Knowledge Management (pp. 391 - 402). Reading, UK: Academic Conferences Limited.

Guerrero Ramírez, Sandra; Ramos-Salinas, N. M.; Muriel Amezcua, Vanesa, (2014) El impacto de los factores tecnológicos (TIC s ) sobre el proceso de gestión del conocimiento en la organización. Editorial Pearson educación, pp. 45-54.

Laud, R.; Thies, P.K. (1997): “Great Expectations: Structuring IT Organizations That Really Deliver". Business Horizons, vol. $40 n^{\circ}$ 4, pp. 25-36.

López Trujillo, M.; Marulanda Echeverry, C. E.; Agudelo Ramírez, J. C.; (Mayo-Septiemre, 2010) Una propuesta sobre buenas prácticas en gestión del conocimiento. Revista Virtual Universidad del Norte, (30), pp 1-25
Ma, Z. \& Yu, K. (2010). Research paradigms of contemporary knowledge management studies: 1998-2007. Journal of Knowledge Management. (14) pp. 175-189.

Macau, R. (2004). TIC: ¿Para qué? (Funciones de las tecnologías de la información y la comunicación en las organizaciones). Revista de Universidad y Sociedad del Conocimiento 1 (1) pp. 1-12

Martínez Carballo, N. (2006) Gestión del conocimiento: Aprendizaje individual versus aprendizaje organizativo. Intangible capital (2) 13 pp. 308-326

Márquez Montoya, S. C.; Ramírez Estévez, M. E.; Losada Losada, M. C. (2007) La gestión del conocimiento, una herramienta imprescindible en la investigación. Ciencia en su PC (5) pp. 3-15

Mata Ordaz de B, Y. V.; Pesca de Acosta, C. A. (2011) La gestión del conocimiento en las universidades como baluarte organizacional. InterSedes: Revista de las Sedes Regionales 12 (23), pp. 56-73.

Nava, M., Esis, E., \& Naval, R. (2011). Capital intelectual: El recurso más valioso. Multiculturalidad: Su análisis y perspectivas a la luz de sus actores, clima y cultura organizacional prevalecientes en un mundo globalizado, 8-27.

Nonaka, Ikujiro y Takeuchi Hirotaka (1999). La organización creadora del conocimiento. Mexico: Oxford University Press.

Nonaka, I. \& Takeuchi, H. (1995), The Knowledge-Creating Company: How Japanese Companies Create the Dynamics of Innovation, Oxford University Press, Inc.

Ramos-Salinas, N.M.; González_Sosa E. (2013). Evolución y Perspectivas de la organización en la Gestión Tecnológica: El impacto de los 
Sistemas de Información. La Gestión Tecnológica y los Sistemas de Información pp. 35-61.

Rakoto, H. (2004), Intégration du retour d'expérience dans les processus industriels : application à Alstom Transport, Thèse de doctorat, Institut National Polytechnique de Toulouse (INPT), France.

Saldarriaga Ríos, J. G.; (2013) Responsabilidad social y gestión del conocimiento como estrategias de gestión humana. Estudios Gerenciales. 29 (126), pp. 110-117.

Senge, P. (1992). La quinta disciplina: El arte y la práctica de la organización abierta al aprendizaje. Barcelona: Granica.

Senge, P. (2012). La quinta disciplina: El arte y la práctica de la organización abierta al aprendizaje. México: Ed. Granica
Scheel, C., Rivera, A. (2009). Utilización de las TIC y su impacto en la competitividad de las empresas latinoamericanas. Universidad y Empresa 8 (16) pp 71-93

Soler M (2011), Caridad Conocimiento médico y su gestión. Revista Habanera de Ciencias Médicas. 10 (4), pp. 505-512.

Smith Cayama, H.; Hurtado Smith, M. (2012) Gestión del conocimiento desde el diseño organizativo. Multiciencias. 12, pp. 151-157

Tseng, C.H., Tansuhaj, P., Hallagan, W., and McCullough, J. (2007) Effects of firm resources on growth in multinationality, Journal of International Business Studies, 38, 961-974.

Valhondo, D. (2003). Gestión del conocimiento: del mito a la realidad. México: Díaz de Santos. 